\begin{tabular}{l}
$\begin{array}{c}\text { XV Encontro Nacional de Tecnologia do } \\
\text { Ambiente COnstruído }\end{array}$ \\
ENTAC 12,13 E 14 DE NOVEMBRO DE 2014 | MACEIÓ | AL \\
\hline
\end{tabular}

\title{
HÁBITATS SOSTENIBLES COMO ESTRATEGIA PARA EL USO RACIONAL Y EFICIENTE DE LA ENERGÍA EN LAS ZNI DE COLOMBIA
}

\author{
BEDOYA TORRES, Sebastian (1); ROMERO HERNÁNDEZ, Antonio José (2); \\ DELGADO JIMENEZ, Jeison Alejandro (3) \\ (1) Universidad Nacional de Colombia, e-mail: sbedoya@unal.edu.co, (2) Universidad Nacional de \\ Colombia, e-mail: aromero@unal.edu.co, (3) Universidad Nacional de Colombia, e-mail: \\ jadelgad@unal.edu.co
}

\begin{abstract}
RESUMEN
Las zonas no interconectadas (ZNI) son municipios, corregimientos y localidades que no están interconectadas al sistema energético central en Colombia. Estas localidades ubicadas principalmente en entornos rurales se caracterizan por la diversidad cultural, ambiental, constructiva y tecnológica; diversidad que contrasta paradójicamente con el desarrollo deficiente de hábitats que no responden de manera funcional, sino a imaginarios asociados al estatus que representa el uso o no de ciertos materiales. El proyecto tuvo como objetivo integrar mediante experiencias constructivas, el conocimiento teórico del diseño bioclimático, los procesos y materiales de origen industrial, el saber ancestral en el uso de los materiales locales, las técnicas constructivas tradicionales y la concepción misma del espacio que responden a características sociales y ambientales de cada localidad. Se realizaron estudios previos de cada zona donde se hizo una recopilación de materiales propios del lugar, técnicas constructivas tradicionales y hábitos culturales para determinar el tipo de prototipo a ser construido. En paralelo, fueron registrados datos climáticos para entender el comportamiento fenomenológico del lugar e integrarlo al diseño de los prototipos. En total el proceso de investigación, diseño, ejecución y monitoreo de los prototipos se desarrolló durante un periodo de más de 3 años; periodo durante el cual hubo un seguimiento constante en cada una de las etapas de desarrollo que permitió entender tanto la maduración de los materiales y sistemas constructivos en el tiempo como la apropiación de estos por las comunidades objeto de estudio.
\end{abstract}

Palabras-clave: Zonas No interconectadas, Desarrollo Sostenible, Bioclimática, Eficiencia Energética.

\begin{abstract}
The Unconnected areas (ZNI in Spanish) are townships that are off the Colombian energy system grid. These localities are mainly in rural surroundings, characterized for high cultural, environmental, constructive and
\end{abstract}


technological diversity, which is in contrast with low habitat developments, and responses, not to the environmental realities but external desires of "quality of life". The project aim was to integrate the different construction methods and cultural inheritance. Enhance the quality with industrial source processes and materials, based upon theoretical knowledge of sustainable design, bearing the ancestral knowledge about the use of local material, traditional construction techniques and space design to respond to specific social and environmental characteristics. In each area, previous tests and information were collected for the prototype design. In addition, environmental data was measured to be applied in the design. All the process took place in a 3 years lapse, with constant monitoring to understand the materials response, construction system behavior and community appropriation.

Key words: Unconnected areas, sustainable development, bioclimatic, energy efficiency.

\section{INTRODUCCION}

En Colombia las Zonas No Interconectadas (o aisladas), ZNI, corresponden a aquellas áreas del país, urbanas o rurales, que no reciben servicio de energía eléctrica a través del Sistema Interconectado Nacional, SIN, y cuya interconexión, no es económicamente factible. El servicio de energía en estas zonas se caracteriza por la baja oferta de servicios energéticos primarios y secundarios, baja cobertura, reducido número de horas de prestación del servicio y bajos niveles de calidad, entre otros. Adicional a estos aspectos, la baja densidad de la población hace difícil la prestación del servicio (UPME, 2000).

Gran parte del área que comprende las ZNI, corresponde a climas cálido-húmedos que, debido a las altas concentraciones de humedad y las elevadas temperaturas, supone grandes consumos energéticos para alcanzar niveles aceptables de confort térmico para quienes las habitan. La pequeña diferencia que existe entre el exterior y el interior de los espacios, disminuye la posibilidad de perder calor en las edificaciones. Adicionalmente, las altas concentraciones de humedad impiden que el cuerpo humano libere calor a través del sudor, pues el aire se encuentra saturado y no puede absorber más humedad (ASHRAE 55:2004). El sudor acumulado en la piel y la alta temperatura del aire producen sensaciones de poco confort, haciendo que las personas alteren su estado de ánimo y disminuyan su disposición para realizar actividades (TRUJILLO, ARROYAVE, 2002).

En estas regiones, las construcciones deben disminuir la ganancia de calor proveniente del sol, al mismo tiempo que deben disipar el calor producido internamente (BITTENCOURT, CÂNDIDO, 2006). Para disminuir la ganancia de calor a través de la irradiación solar, se recomienda que las fachadas de las construcciones de estos climas estén sombreadas adecuadamente y que utilicen superficies con baja absortancia solar. Además de esto, se debe maximizar la ventilación con el fin de compensar las necesidades de confort térmico.

Al incrementar la velocidad del aire, se puede aumentar la evaporación en la superficie de la piel produciendo una sensación de refrescamiento. Por ejemplo, en un ambiente con velocidades de aire de $0.8 \mathrm{~m} / \mathrm{s}$ con humedad relativa de $60 \%$, la temperatura de bulbo seco podría estar $2^{\circ} \mathrm{C}$ por encima del límite superior de la zona de confort térmico debido a la compensación que puede obtenerse con esa velocidad (ALLARD, SANTAMOURIS, 1998). Esto significa que los ocupantes pueden estar en buenas condiciones de confort térmico, aun cuando se tengan temperaturas más altas, gracias al aumento de la velocidad del aire. 


\section{OBJETIVO}

El proyecto tuvo como objetivo integrar mediante experiencias constructivas, el conocimiento teórico del diseño bioclimático, los procesos y materiales de origen industrial, el saber ancestral en el uso de los materiales disponibles, las técnicas constructivas tradicionales y la concepción misma del espacio que responden a características sociales y ambientales de cada localidad.

\section{METODO}

Entendiendo que los grandes retos en lo que a generación de confort se refiere están en los climas cálido-húmedos fueron elegidas 6 comunidades de las ZNI para realizar un ejercicio de integración cultural y tecnológica a través de procesos constructivos; el proyecto tuvo una duración de más de 3 años, periodo durante el cual hubo un seguimiento constante en cada una de las etapas de desarrollo, permitiendo entender tanto la maduración de los materiales y sistemas constructivos en el tiempo, como la apropiación de estos por las comunidades objeto de estudio.

La estructura a través de la cual se realizó el proyecto comprendió 4 etapas: Caracterización, diseño, implementación y monitoreo; las cuales permitieron desarrollar el concepto, el modelo, el prototipo y con ello aproximarse de manera integral a la problemática del hábitat, la eficiencia energética, y el uso de los diferentes materiales en las ZNI de Colombia.

Las comunidades de las Zonas No Interconectadas en las cuales se realizó el proyecto fueron: Isla Fuerte en el departamento de Bolívar, El Totumo y Murindó en el departamento de Antioquia, Titumate y Pizarro en el departamento de Chocó y Nazareth en el departamento de la Guajira.

\subsection{Caracterización}

Esta etapa permitió tener un primer acercamiento a los procesos involucrados en el hábitat de cada una de las zonas (Ver Figura 1). El proyecto, incluyó visitas a cada una de las poblaciones, las cuales representan tres condiciones ambientales: Cálido-seco, cálidohúmedo continental y cálido- húmedo insular. Cada uno de estos representaba formas distintas de habitar y apropiarse de los materiales del territorio y en torno a ello se desarrolló la etapa de caracterización. Las premisas principales fueron: conocer los materiales que se utilizaban, las tecnologías constructivas predominantes y los procesos de adaptación y consumo de los recursos de cada lugar. Durante esta etapa un grupo interdisciplinar, realizó visitas de campo que permitieron estudiar de manera directa las dinámicas sociales, ambientales, tecnológicas y energéticas de cada lugar. 


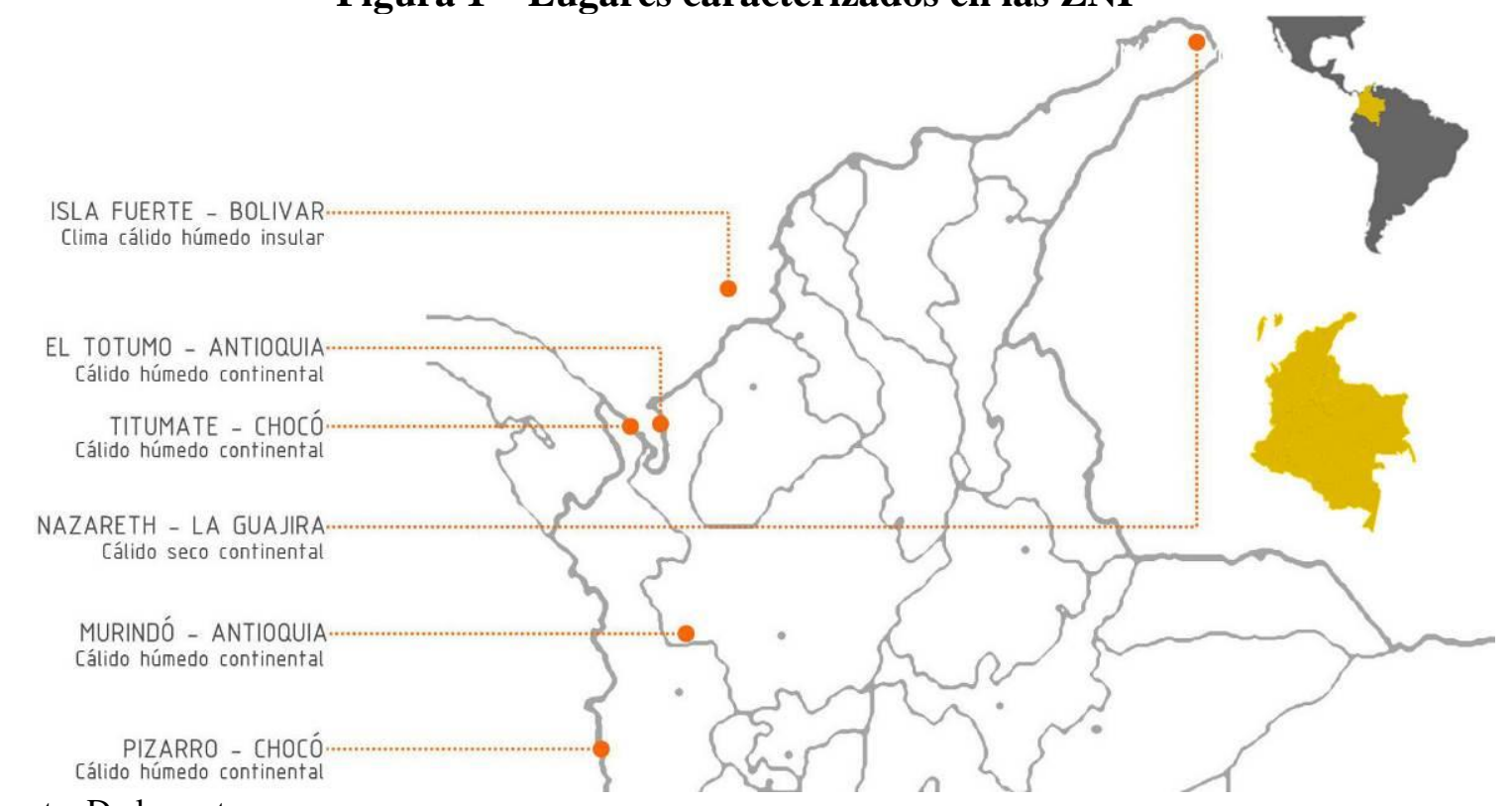

Fuente: De los autores

Las actividades realizadas se clasificaron así:

- Componente ambiental: Se orientó a documentar como se daban los procesos de Manejo de residuos, gestión de agua y alimentos.

- Componente socio-económico: Estructura social, movilidad, estructura familiar, actividades económicas y nivel de ingresos.

- Componente arquitectónico: Materiales, sistemas constructivos, patologías de los materiales, configuraciones espaciales, y el monitoreo a través de sensores para medir humedad relativa y temperatura en distintas tipologías de vivienda, en distintas orientaciones con respecto al sol, corrientes de viento predominantes, barreras vegetales y condiciones topográficas.

Durante las salidas de campo fueron documentados los rituales cotidianos que se establecen como forma de adaptación según las condiciones culturales, económicas y ambientales de cada lugar (Ver figura 2). La información y los estudios realizados durante esta etapa se constituyeron en la línea base para el desarrollo de las etapas posteriores.

Figura 2 - Imágenes Nazareth - Alta Guajira

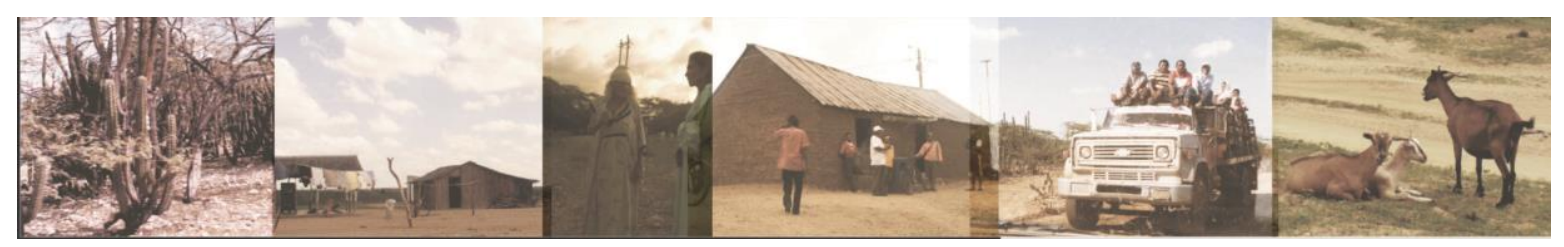

Fuente: De los autores 


\subsection{Diseño}

En el proceso de diseño fue incorporada la información levantada en campo que permitió generar los diseños según las particularidades de cada lugar, la línea de desarrollo se estableció integrando el conocimiento teórico del diseño bioclimático, los procesos y materiales de origen industrial, y las tecnologías constructivas que se identificaron en la línea base. La base teórica del diseño bioclimático vinculó conceptos de inercia térmica en los materiales y fenómenos de convección como estrategia en la ventilación, ubicación con respecto al sol, al viento y la vegetación (Ver figura 3). Adicionalmente se consideró incluir en el diseño materiales y técnicas ancestrales usadas en la implementación de cerramientos y cubiertas propias de cada localidad.

\section{Figura 3 - Proceso de diseño}

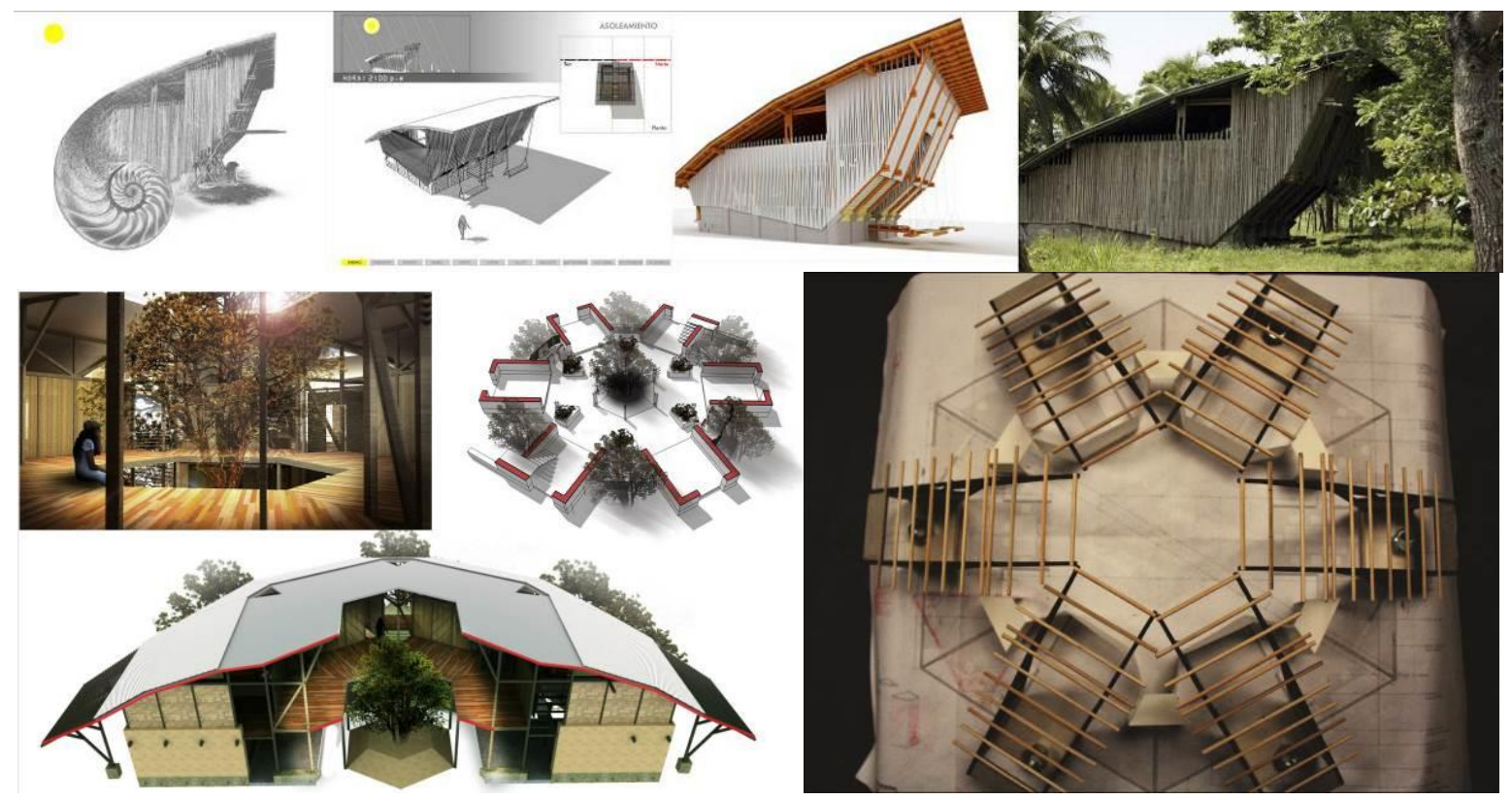

Fuente: De los autores

\subsection{Implementación}

Una vez generados los diseños se definieron las estrategias que posibilitarían la implementación de los prototipos diseñados en cada localidad. Estas estrategias consistían en factores logísticos como transporte, disponibilidad de recursos, mano de obra y conocimiento especializado (saberes locales), además de tiempos de ejecución, y las condiciones climáticas dado el difícil acceso en épocas de invierno a algunas localidades. Estas estrategias se adaptaron prestando especial atención a las particularidades de cada entorno y la conciliación de las técnicas y materiales de origen industrial (Ver figura 4), con la mano de obra y materiales disponibles en cada zona al momento de la construcción, especialmente cañas, fibras y bejucos por sus propiedades aislantes y abundancia. 
Figura 4 - Proceso de implementación
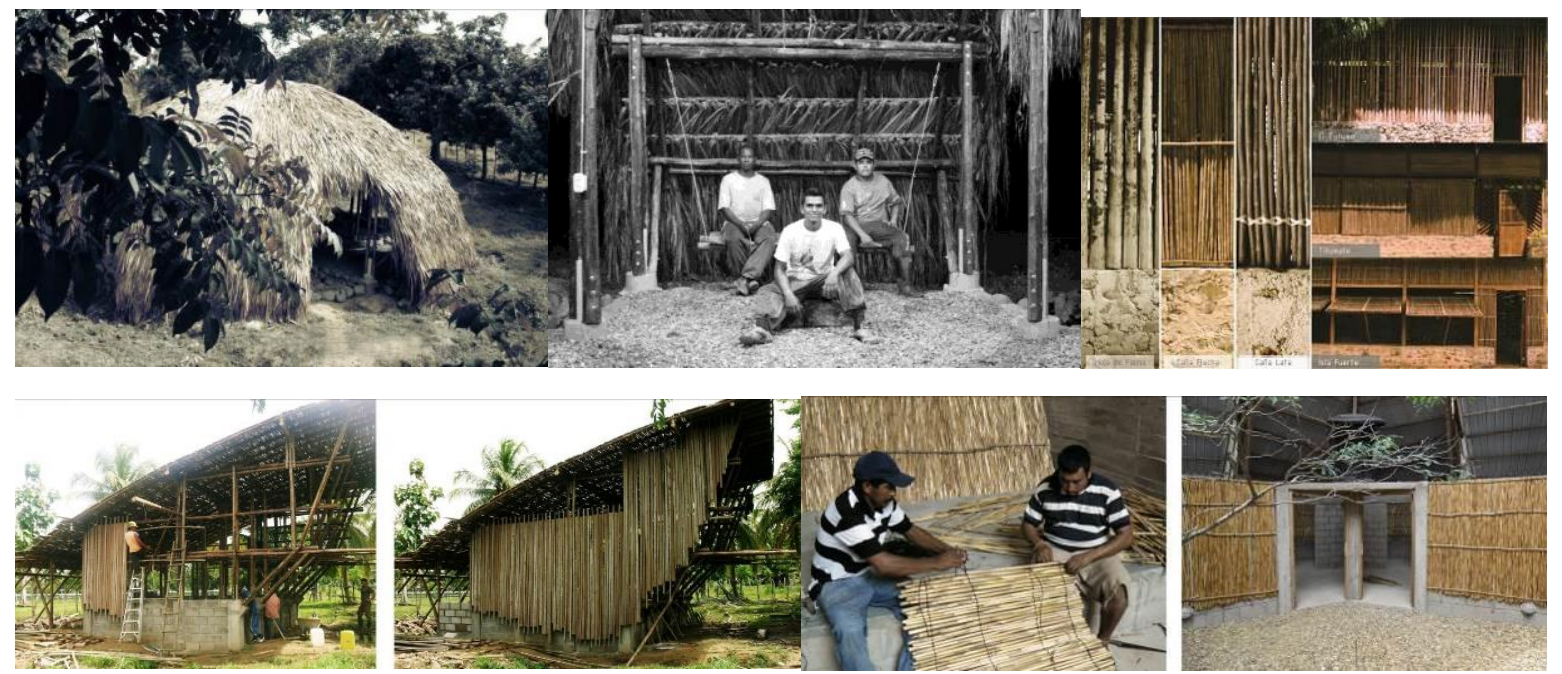

Fuente: de los autores

\subsection{Monitoreo}

Los prototipos implementados quedaron insertados en el territorio bajo las particularidades del mismo y fueron objeto de estudio a través de un seguimiento constante. Durante esta etapa fueron realizadas visitas periódicas a cada uno de los lugares para documentar tanto la maduración de los materiales y sistemas constructivos utilizados, como la apropiación de los espacios por parte de los habitantes de cada localidad. Durante esta etapa fueron instalados sensores que midieron humedad relativa y temperatura en cada uno de los espacios implementados durante un periodo de un año (Ver figura 5).

\section{Figura 5 - Monitoreo humedad relativa y temperatura}

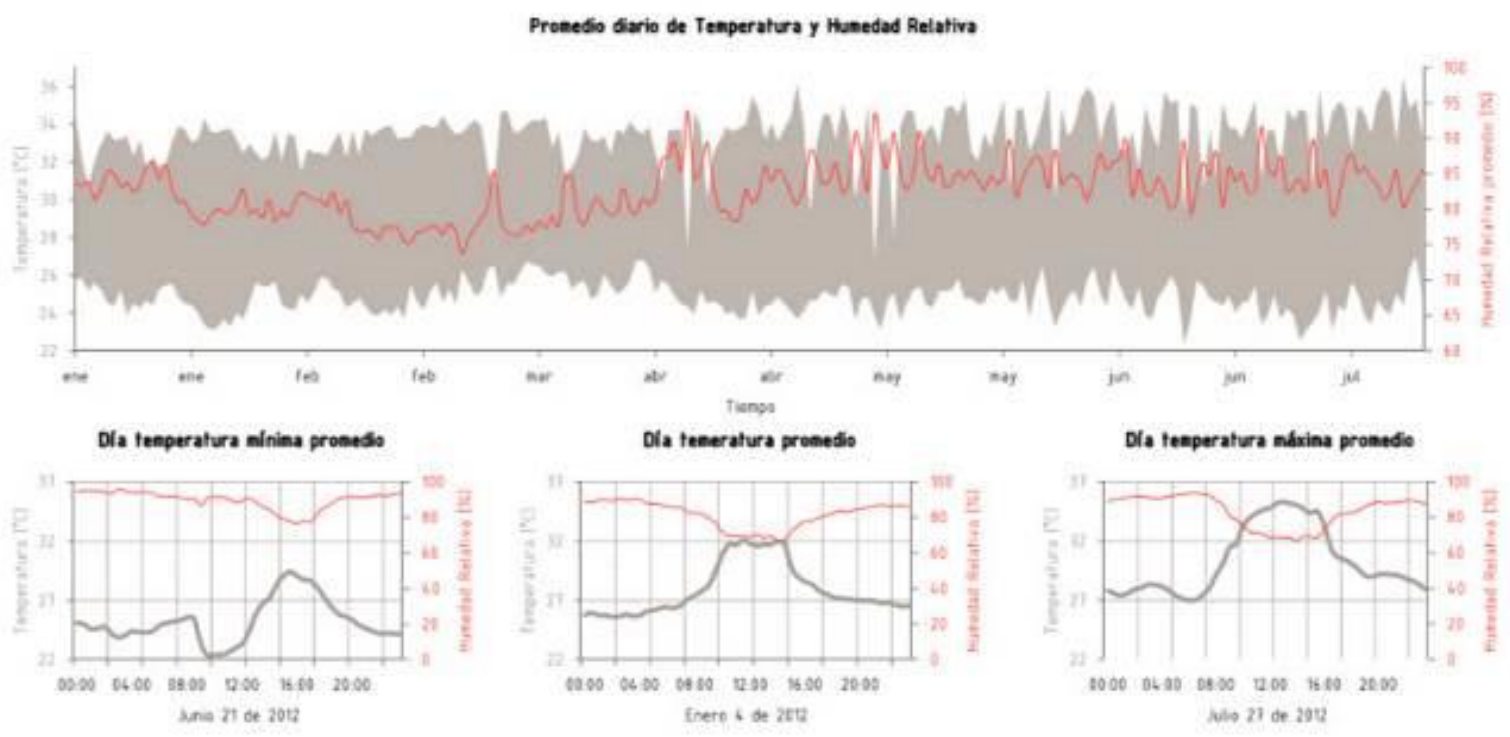

Fuente: de los autores 


\section{RESULTADOS}

Como resultado de esta investigación fueron diseñados, implementados y monitoreados 7 prototipos habitables desarrollados así: 2 prototipos en clima cálido húmedo insular (Isla Fuerte), 4 en clima cálido húmedo continental (El Totumo y Titumate) y 1 en clima cálido seco continental (Nazareth), (Figura6).
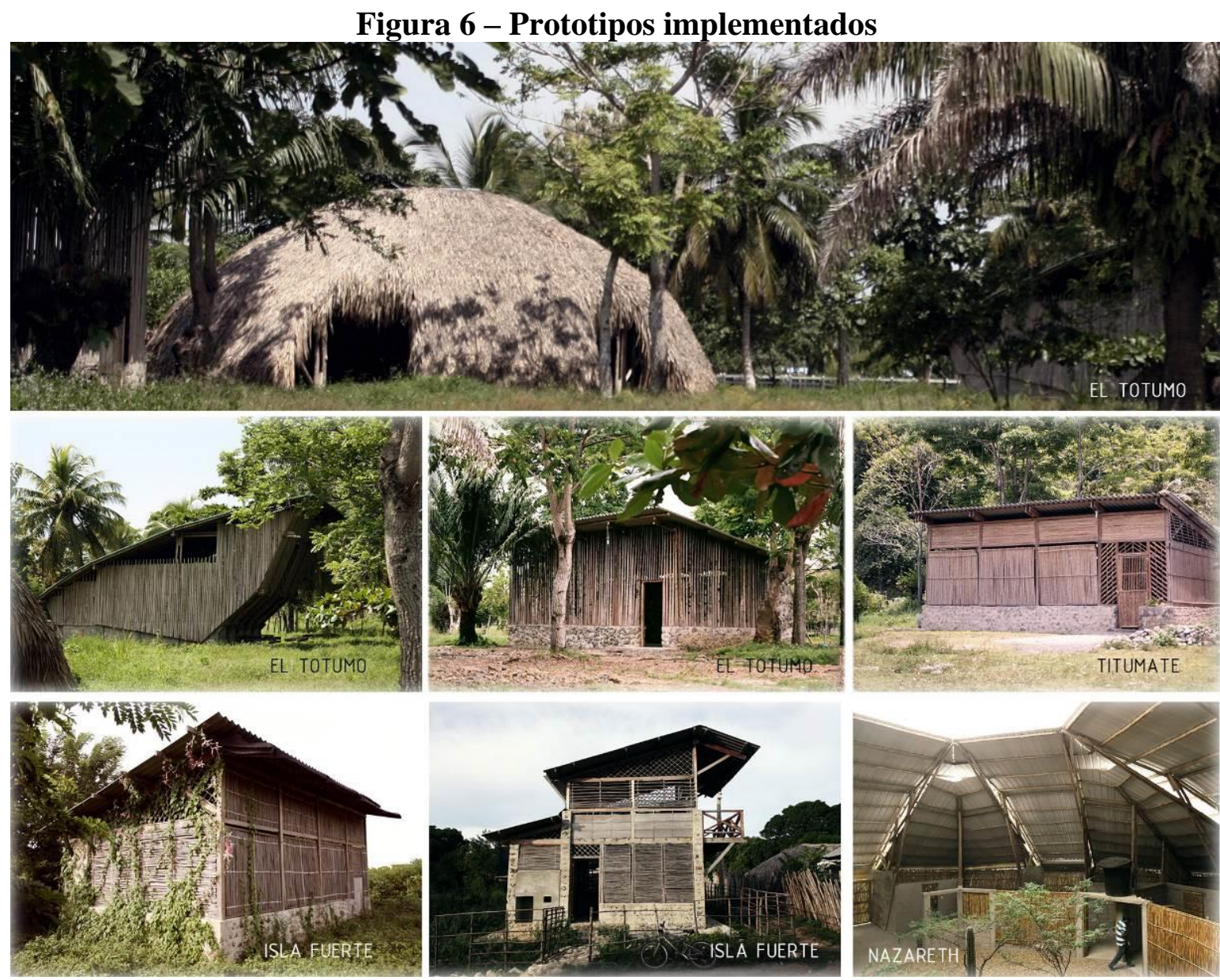

Fuente: De los autores

El desarrollo metodológico, permitió un conjunto de aprendizajes en la relación, teoría y prácticas empíricas a través de la integración de tecnologías industriales y ancestrales. Este conjunto de aprendizajes puede resumirse en función del acercamiento de los diferentes saberes y la conciliación de los mismos, mediante la implementación de soluciones que responden a problemáticas de orden local, pensadas tanto desde las dinámicas urbanas como desde las realidades y necesidades rurales. Es importante resaltar que, además de las intervenciones puntuales como los prototipos implementados, la gran enseñanza está representada en el conocimiento acumulado durante el desarrollo del proyecto, en la interacción a través de una estructura colaborativa entre el universo urbano y rural, académico y estatal, interacciones afortunadas que posibilitaron los mecanismos de acción para el desarrollo de soluciones sostenibles que mejoren la calidad de vida de las poblaciones más necesitadas. 


\section{CONSIDERACIONES FINALES}

Si bien, el desarrollo de soluciones apropiadas en la vivienda, dan respuesta a unas necesidades específicas de habitabilidad en los entornos objeto de estudio, no son el punto final del proceso, la solución debe ser mucho más dinámica, A estos procesos de generación del hábitat deben sumarse estrategias para la conservación del entorno a una escala territorial mayor que permita mejorar la gestión de alimentos, de agua y generación de energías alternativas, además de promover prácticas que las hagan cultural y ambientalmente sostenibles.

Mediante el uso correcto de los materiales locales fue posible demostrar a los habitantes de cada localidad la importancia de incorporarlos dentro de las soluciones de vivienda como estrategia para la generación de hábitats cultural y ambientalmente más sostenibles.

Durante la etapa de monitoreo se encontró que la combinación entre los valores de temperatura y humedad relativa se encuentra en su mayoría por fuera de la zona de confort reflejando las exigentes condiciones climáticas en los cuales fueron emplazados todos los prototipos. Debido a la complejidad que implicaba hacer un estudio adecuado de ventilación dentro de la campaña de monitoreo realizada, no fue posible establecer la compensación inducida por el viento en el interior de los prototipos. No obstante, se debe resaltar que el diseño de estos atiende a las recomendaciones indicadas por la carta bioclimática, esto es: mejorar la ventilación, sombrear las fachadas y maximizar la cantidad de viento que entra en cada prototipo mediante el uso de cerramientos que aun al estar cerrados, permitieran el paso del aire.

Los prototipos implementados integraron los componentes ambientales y culturales claves de cada sitio, y posibilitó su inserción como referente constructivo y elemento generador de identidad. A través de testimonios realizados por los habitantes de cada localidad fue posible constatar el nivel adecuado de confort logrado al interior de los prototipos implementados (Figura6).

\section{Figura 6 - Prototipos implementados}
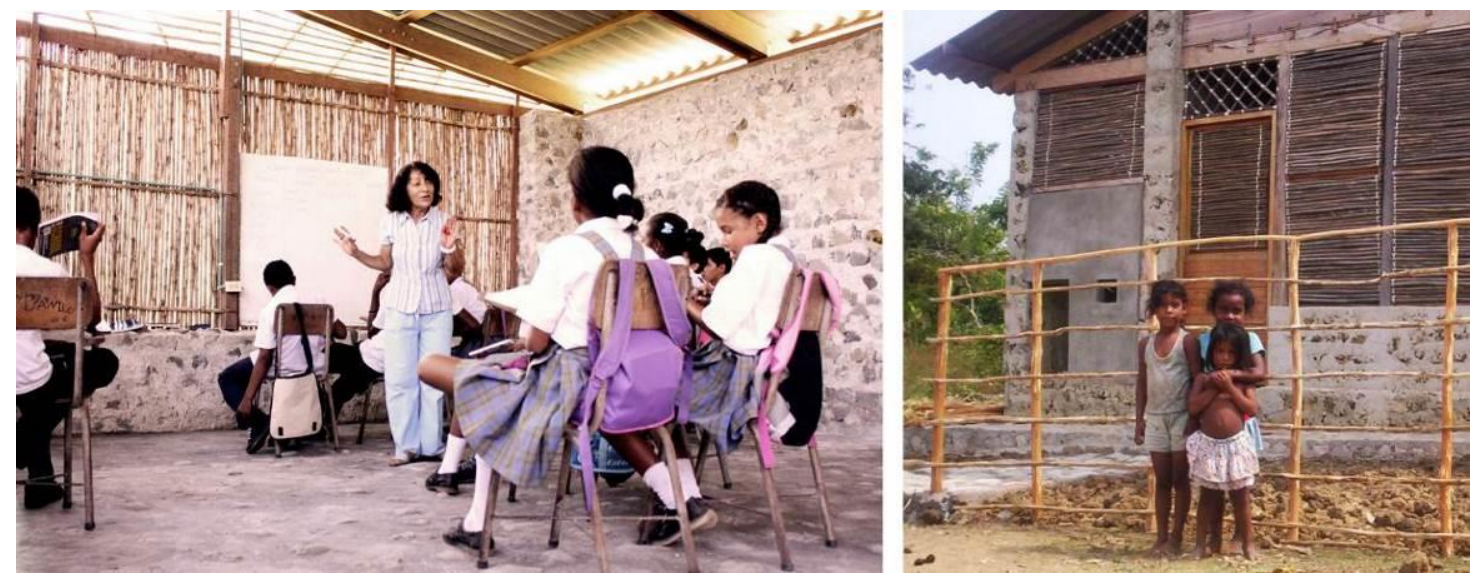

Debido a la abundancia y rápido crecimiento, los materiales usados en los cerramientos no comprometió la disponibilidad del recurso, por otro lado, al tratarse de sistemas constructivos locales posibilitan realizar las reparaciones que puedan ser requeridas para el 
mantenimiento de los prototipos. No existen materiales buenos o malos, siempre dependerá del cómo y dónde sean usados, estos deberán ser evaluados según los requerimientos de cada lugar para establecer jerarquías constructivas según su disponibilidad, duración, desempeño ambiental, y requerimientos espaciales. En el desarrollo de los prototipos fueron usados materiales como cañas fibras y bejucos, que disponiéndolos de manera correcta ofrecieron un buen desempeño además de ser materiales abundantes de rápido crecimiento.

Durante el desarrollo del proyecto hubo muchas enseñanzas para el grupo que lo llevo a cabo, pero quizás la más importante fue entender que aun cuando se comparte una misma realidad global, cada lugar está regido por unas nociones de tiempo y espacio que determinan la manera de interpretarlo e intervenirlo. Deben promoverse procesos que generen una postura diferente frente a la manera en que se interactúa con el entorno, partiendo de la generación de espacios habitables, que se extienden como un elemento dentro del complejo sistema de componentes que permiten la permanencia de las comunidades en una zona determinada.

\section{AGRADECIMIENTOS}

Al instituto de Planificación y Promoción de Soluciones Energéticas del Ministerio de Minas y Energía de Colombia (IPSE). Instituto encargado de desarrollar sistemas de generación alternativos para las ZNI, el cual en convenio con la Universidad Nacional de Colombia sede Medellín posibilitó el desarrollo del proyecto.

\section{REFERENCIAS}

ALLARD, Francis; SANTAMOURIS, Matheos. Natural Ventilation in Buildings: A Design Handbook. London: James and James, 1998.

BITTENCOURT, L.; CÂNDIDO, C. Introdução à ventilação natural. 2 ed. rev. e ampl. Edufal. Maceió, AL 2006.

LABEEE - LABORATÓRIO DE EFICIÊNCIA ENERGÉTICA EM EDIFICAÇÕES. Universidad Federal de Santa Catarina. Programa computacional Analysis Bio, versão: 2.2. Disponible en: http://www.labeee.ufsc.br/downloads/softwares/analysis-bio.

LAMBERTS, Roberto; DUTRA, Luciano; PEREIRA, Fernando O. R. Eficiência energética na arquitetura. São paulo: Pw Editores, 1997. 192 p. Disponible en: http://www.labeee.ufsc.br/publicacoes/livros.

TRUJILLO, Jorge Hernán Salazar; ARROYAVE, Rafael Hernán Atehortua. Construcción de normativas urbanísticas para el ahorro energético en climas tropicales. Medellín: Universidad Nacional de Colombia, 2002.

UPME, Establecimiento de un plan estructural, institucional y financiero, que permita el abastecimiento energético de las zonas no interconectadas, con la participación de las comunidades y el sector privado, in 'Metodología', UPME - Ministerio de Minas y Energía Unidad de Planeamiento Minero Energético, 2000.

ASHRAE. Thermal environmental conditions for human occupancy. (No. De publicación ASHRAE 55:2004), Atlanta, USA 2004. 